\title{
Proapoptotic genes BAX and CD4OL are predictors of survival in transitional cell carcinoma of the bladder
}

\author{
SA Hussain', R Ganesan², L Hiller ${ }^{3}$, PG Murray', MM El-Magraby', L Young' and ND James*,1 \\ 'Cancer Research UK Institute For Cancer Studies, University of Birmingham, Vincent Drive, Edgbaston, Birmingham BI5 2TT, UK; ${ }^{2}$ Department of \\ Pathology, Birmingham Woman's Hospital, Birmingham, UK; ${ }^{3}$ Cancer Research UK Trials Unit, Institute For Cancer Studies, Birmingham, UK
}

Bladder cancer will be diagnosed in more than 54000 people this year in USA, and over 12000 will die of the disease (ASCO online). Bladder cancer, with 12730 new cases in 1997, is the fifth commonest cause of cancer in the UK (Cancer Research UK Fact Sheet). Approximately $20 \%$ of newly diagnosed cases are invasive and, in this group, prognosis is poor with only $60 \%$ of T2 and $40 \%$ of T3 patients surviving 5 years. Patients with more advanced or nodal disease do significantly worse; median survival with metastatic disease for those patients fit enough to enter trials is approximately 13 months (m). Management of locally advanced disease is by surgery or radiotherapy, both of which have significant drawbacks. Neoadjuvant and adjuvant chemotherapy have been tested extensively but to date this approach has not had a significant impact on overall survival (Frieha et al, 1996; Malmstrom et al, 1996; Stockle et al, 1996; International Collaboration of Trialists, 1999). Cisplatinum-based combination chemotherapy forms the mainstay of therapy for relapsed or metastatic disease with response rates of up to $60-70 \%$ reported, but few long-term survivors. Intravesical and systemic chemotherapy are limited in their efficacy in the treatment of bladder cancer patients as they are unable to induce apoptosis in bladder tumour cells. Understanding the apoptotic signals and the cascade of reactions that give prosurvival signals will go a long way in

*Correspondence: Dr ND James, Vincent Drive, Edgbaston, Birmingham BI5 2TT, UK; E-mail: n.d.james@bham.ac.uk

Received 23 July 2002; revised 19 November 2002; accepted 19 November 2002 refining the treatments and will help in future to individualise cancer therapies.

We studied a range of molecular markers to define their roles and their association with other markers to define targets against which we can target appropriate therapies.

$\mathrm{CD} 40$ is a $48 \mathrm{kDa}$ membrane glycoprotein of the tumour necrosis factor receptor (TNFR)/nerve growth factor superfamily, and is expressed on the surface of B lymphocytes, dendritic cells, epithelial and haemopoietic stem cells (Lee et al, 1997), and various tumours. It was first described in 1985 as a bladder tumour antigen (Banchereau et al, 1994) but this was largely overlooked when subsequent investigation revealed its pivotal role in B-cell function, where ligation with CD40 ligand (CD40L or CD154) expressed on activated T-helper cells stimulates cell maturation, proliferation and immunoglobulin class switching. Despite its role as a promoter of B-cell growth, it has been previously shown that, in common with other members of the TNFR family, CD40 stimulation sensitises carcinoma cells in vitro to apoptosis induced by various stimuli including Fas, ceramide and cytotoxic chemotherapy (Eliopoulos et al, 1996).

CD40 ligand (CD40L), also known as CD154, functions as the natural ligand for CD40 (Spriggs et al, 1992; Armitage et al, 1992). It is expressed primarily by on the surface-activated T lymphocytes (Armitage et al, 1992) but has also been found on activated $\mathrm{T}$ platelets (Henn et al, 1998). Interactions between CD40 and CD40L provide critical costimulatory signals that trigger T-lymphocyte expansion (Grewal et al, 1998).

We have previously demonstrated that quantification of the antiapoptotic protein BCL2 in patients undergoing neoadjuvant chemotherapy plus radiotherapy for advanced bladder cancer may 
identify patients who might benefit from neoadjuvant chemotherapy (Cooke et al, 2000). The BCL2 oncoprotein inhibits apoptosis and is overexpressed by many tumours including breast (Hellemans et al, 1995), colon (Bronner et al, 1995), prostate (Krajewska et al, 1996) and tumours of the head and neck (Gallo et al, 1999). By virtue of its biological activity, it may be associated with a poor prognosis, with resistance to current treatment modalities including radiotherapy, for example, in cervical (Harima et al, 1998) and prostate cancer (Apakama et al, 1996). The data available to date, describing the role played by BCL2 in transitional cell carcinoma (TCC) of the bladder, have been both limited and conflicting, with some studies showing an association with a lower tumour grade (King et al, 1996) and less aggressive phenotype (Shiina et al, 1996). Others have shown the reverse, with expression being greater in higher-stage and higher-grade tumours, resulting in increased frequency of disease recurrence and higher disease progression rates, leading to shortened survival (Ye et al, 1998; Pollack et al, 1997; Kong et al, 1998). It is likely that these conflicting results arise because of different interactions between the various treatment modalities and apoptotic pathways. Many of the effects of BCL2 depend on its ratio to BAX as well as on its absolute level. The proapoptotic $\mathrm{BH}-123$ proteins share three of the BCL2 homology $(\mathrm{BH})$ domains with the antiapoptotic proteins and include BAX, BAK, and BOK. BAX plays a central role in regulation and commitment to apoptosis. In order to further examine these phenomena, we therefore set out to investigate the linked genes BAX and BCL2 expression in the same group of patients, and to examine whether these discrepant results are explained by variations in other genes involved in the same pathways.

We therefore proceeded with immunohistochemical analysis of these anti-apoptotic and pro-apoptotic genes to look for correlation between themselves and the effects of their expression on clinical outcome and survival. We report the expression of antiapoptotic BCL2, proapoptotic BAX, CD40, CD40L and accumulation of $\mathrm{P} 53$ in a series of patients with transitional cell carcinoma of the bladder.

\section{MATERIALS AND METHODS}

Expression of genes was carried by immunohistochemical analysis of the paraffin tissue archive. The tissue collection and the study had full local research ethics approval. Ninety-four patients with TCC of the bladder were identified from hospital records (74 males, 20 females, median age 67 years, range $32-89$ years). Of these, 45 had noninvasive tumours and were included in two clinical trials for primary and recurrent Ta and T1 tumours, and 49 had invasive tumours, of which 26 patients were treated in a pilot study of chemoradiation in muscle-invasive bladder cancer (Hussain et al, 2001) and 23 patients were treated in a radiotherapy study examining the role of neoadjuvant cisplatin for muscleinvasive tumours (Wallace et al, 1991). Histological classification of the patients' tumours was obtained from the original hospital/ trial pathology records and was according to the Union Internationale Contre le Cancer (UICC) TNM system, 1997. The archived tissue specimens were retrieved, all of which had been routinely fixed in $10 \%$ saline-buffered formalin and embedded in paraffin wax. One representative tissue block was chosen for each patient on the basis of gross specimen morphology, and serial $5 \mu \mathrm{m}$ sections were cut from it and mounted onto microscope slides. One section from each specimen was then immunostained for BCL2, BAX, CD40, CD40L and P53, plus a specimen for routine haematoxylin and eosin staining.

\section{Staining procedure}

Tissue sections were deparaffinised in xylene, washed in alcohol and rehydrated in $0.05 \mathrm{M} \mathrm{pH} 7.6$ phosphate-buffered saline (PBS). Antigen retrieval was performed in a $700 \mathrm{~W}$ microwave oven in $0.01 \mathrm{M} \mathrm{pH} 5.8$ citrate buffer solution for $30 \mathrm{~min}$ as optimised in preliminary studies. The sections were cooled to room temperature in PBS, the primary antibody applied and incubated in a moist chamber at room temperature for $1 \mathrm{~h}$. The primary antibodies and concentrations used were as shown in Table 1. The secondary staining and tertiary staining was performed using DAKO strept ABC complex (DAKO Ltd, Cambridgeshire, UK). Secondary and tertiary antibodies were applied and incubated in a moist chamber at room temperature for $30 \mathrm{~min}$. Sections were counterstained in Mayer's haematoxylin and mounted in aqueous mountant (Shandon, UK). Sections of tonsil were used as positive control for each staining run. Substituting primary antibody with PBS created negative controls. Tissue lymphocytes acted as an additional internal control for the CD40- and BCL2-stained sections. Evaluation of immunostaining (Table 2) was performed on two separate occasions by one observer (SAH) and once by a pathologist (RG), both of whom were blinded to any other data. The whole of each section was subjectively assessed under light microscopy. There was one score for the strength of staining (absent, weak or strong, respectively, as compared with the positive controls) and one score for the percentage of tumour stained (absent, focal/patchy or diffuse). Staining of mitotic figures was ignored and only nuclear staining was regarded as positive for P53. Sections where intra or interobserver error occurred for either of the scores were reviewed again by a pathologist (RG or MM) and assigned a score that dictated which of the two original scores was recorded. In the event of all three pairs of scores differing, a consensus score was agreed upon after examination under a multiheaded microscope. Tumours were classified into five patterns of expression: tumour negative; weak diffuse (WD); weak focal (WF); strong diffuse (SD); or strong focal (SF). Of the 94 specimens, satisfactory staining was achieved in 86 cases $(91.5 \%)$. In cases where staining was not optimal for evaluation, they were

Table 2 Evaluation of staining

\begin{tabular}{lll}
\hline & BCL2, BAX, CD40, CD40L & P53 \\
\hline Diffuse & Confluent positivity & $>30 \%$ of nuclei \\
Focal & Nonconfluent islands of positivity & $<30 \%$ of nuclei \\
Strong & Intensely brown & $\begin{array}{l}\text { Intensely brown } \\
\text { Weak }\end{array}$ \\
\hline
\end{tabular}

Table I Antibodies used

\begin{tabular}{lllll}
\hline Antibody & Code & Source & Dilution & Supplier \\
\hline BCL2 & (C-2):Sc-7382 & Mouse monoclonal antibody & $1: 10$ & Santa Cruz, CA, USA \\
BAX & P-19:Sc-526 & Rabbit purified polyclonal antibody & $1: 20$ & Santa Cruz, CA, USA \\
CD40 & (N-16):Sc-974 & Rabbit polyclonal antibody & $1: 100$ & Santa Cruz, CA, USA \\
CD40L & (C-20):Sc-978 & Rabbit polyclonal antibody & $1: 100$ & Santa Cruz, CA, USA \\
P53 & (Bp 53-12):Sc-263 & Mouse monoclonal antibody & $1: 20$ & Santa Cruz, CA, USA \\
\hline
\end{tabular}


left out of the study. In some cases only normal or inflammatory tissue was identified with no evidence of transitional cell carcinoma. In the remaining 81 out of the $94(86 \%)$ cases stained for BAX, 83 out of the $94(88 \%)$ for BCL2, 79 out of the $94(84 \%)$ for CD40, 79 out of the $94(84 \%)$ for CD40L and 78 out of the 94 $(83 \%)$ for P53 contained foci of malignancy.

\section{Statistical methods}

Intercorrelations between the laboratory measures were explored by use of Fisher's exact tests and Correspondence Analysis (Greenacre, 1984), an exploratory technique used to analyse multi-way tables. Survival times were calculated as the date of primary tumour diagnosis to date of death, or date of censor if alive. Survival curves were constructed using the method of Kaplan and Meier (1958), and the log-rank test (Peto et al, 1977) was used to assess any differences between patient and tumour characteristics. Cox proportional hazards analysis (Cox, 1972) was also performed, using a 5\% entry and exit criterion, to determine important independent prognostic factors of survival.

Statistical analysis was carried out independently using SAS statistical software (SAS Institute, Cary, NC, USA).

\section{RESULTS}

\section{Intercorrelation between laboratory measures}

Fisher's exact tests showed the BCL2 marker to be independent of all others $(P>0.05)$. P53 was also shown to be independent of all other markers, except a borderline dependence with BAX $(P=0.04)$. BAX, CD40 and CD40L were all significantly associated with each other $(P \leqslant 0.05)$. Correspondence analysis confirmed the above by showing a separate tight clustering of the expression levels of BAX, CD40 and CD40L, which were also separate from the two distinct levels of both BCL2 and P53. This indicates that BAX, CD40 and CD40L vary together and thus explain similar characteristics about patients. BCL2 and P53 are shown as individual markers dissimilar to all others thus measuring different things.

Analysis of the individual subgroups (negative, WF, WD, SF, SD) demonstrated statistically significant interactions between all subgroups of expression for BAX, with strong positivity with a diffuse pattern of BAX expression as an independent prognostic marker of survival $(P=0.0001)$. However, since focality of expression was a relatively subjective observation dependent on variable factors such as the even distribution of antigen at the time of staining, we grouped the results into better definable groups based only on the strength of staining. Despite ignoring the pattern of staining, BAX remained an independent marker of prognostic significance of survival $(P=0.0002)$. Patients with tumours overexpressing BAX had a median survival of $110 \mathrm{~m}$ as against a group of patients with weak expression or no expression of BAX who had a median survival of $18 \mathrm{~m}$ (Figure 1). Similar considerations applied to CD40L and so, for practical purposes, cases were then grouped as either positive (strong) for (SF or SD) expression or negative (weak) for (negative, WF or WD) expression, as this gave the most easily clinically applicable scoring system. There were no significant differences seen for expression of CD40, P53 or BCL2, but the same grouping system was applied to these groups in the interests of consistency of analysis.

The median survival for all patients from the day of diagnosis of primary tumour was $80 \mathrm{~m}$. There were statistically significant effects according to expression levels of BAX $(110 \mathrm{~m} v s 18 \mathrm{~m}$, $P=0.0002$ ) (Figure 1) and CD40L ( $95 \mathrm{~m} v s 45 \mathrm{~m}, P=0.04$ ), with overexpression of CD40L leading to better survival (Figure 2). For CD40, median survival by subgroup was positive $110 \mathrm{~m}$ and negative $45 \mathrm{~m}, P=0.12$ (Figure 3 ); for BCL2, positive $44 \mathrm{~m}$ and

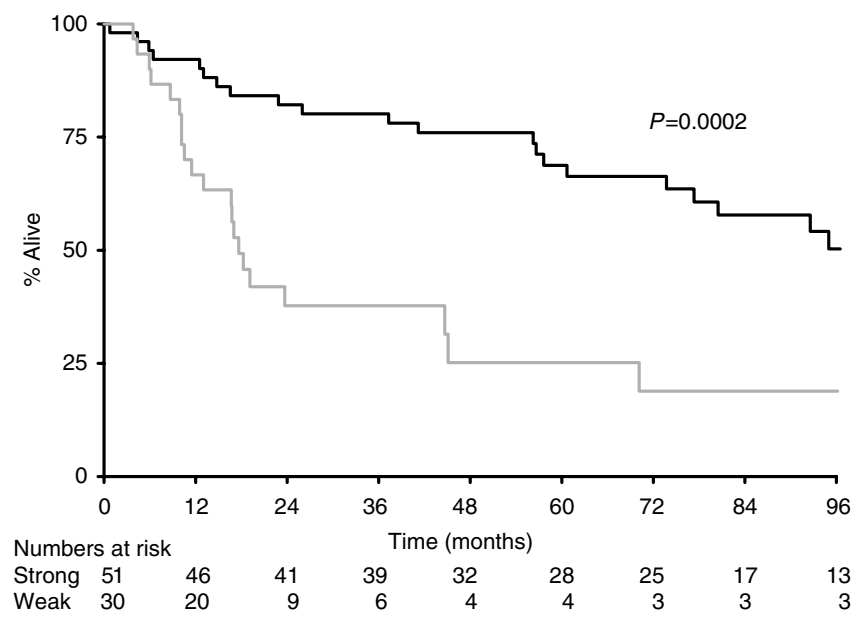

Figure I Survival by BAX ( - strong; - weak).

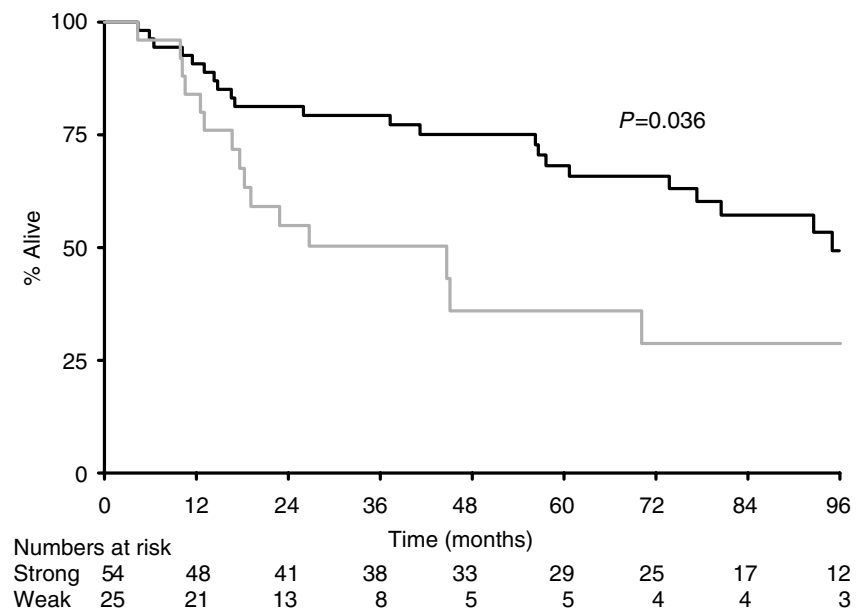

Figure 2 Survival by CD40L ( - strong; - weak).

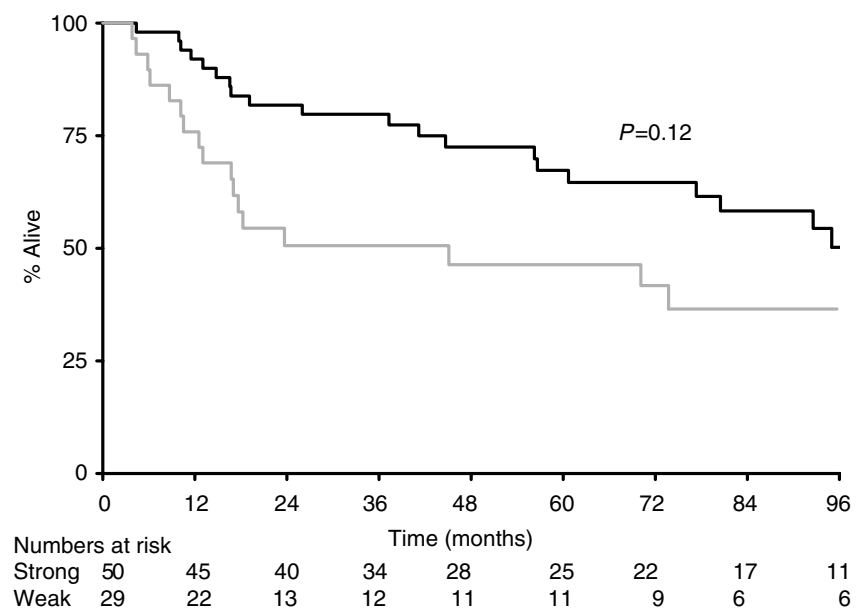

Figure 3 Survival by CD40 (- strong; - weak).

negative $74 \mathrm{~m}, P=0.64$ (Figure 4). For P53, the results were again not significant with median survivals of $80 \mathrm{~m}$ for positive and $45 \mathrm{~m}$ for negative $P=0.58$ (Figure 5). As would be expected, there were significant associations between tumour stage $(\mathrm{Ta} / 1$ vs $\mathrm{T} 2$ vs $\mathrm{T} 3$ vs T4 with median survivals of $128,23,19$ and $27 \mathrm{~m}$, respectively, 


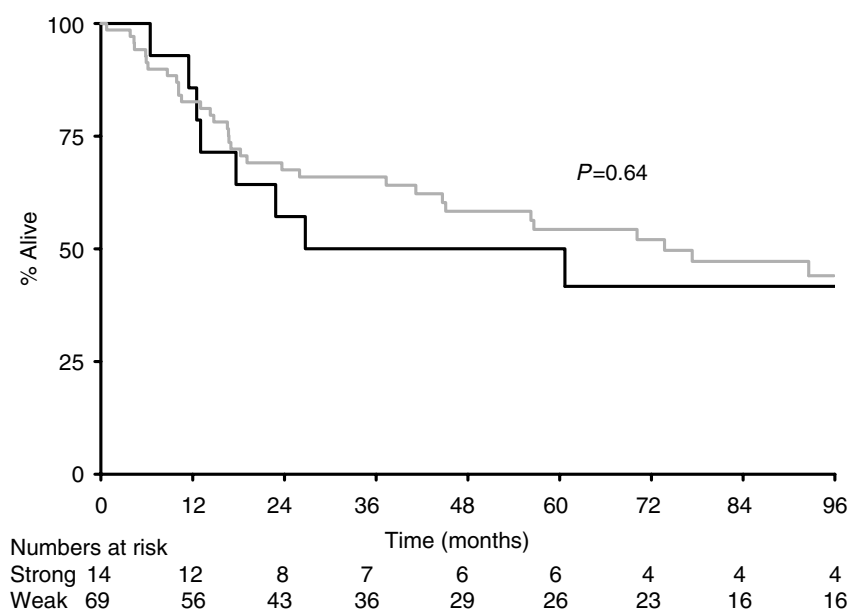

Figure 4 Survival by BCL2 (— strong; — weak).

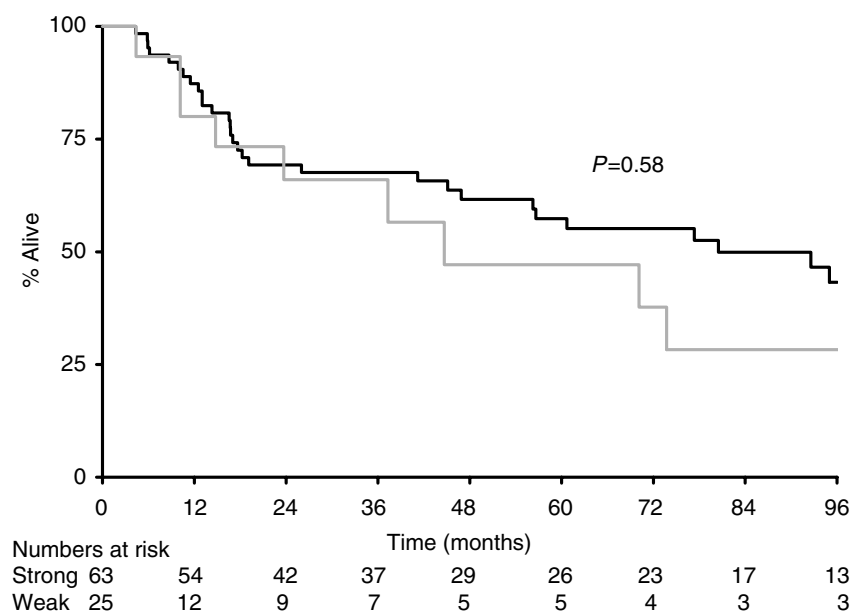

Figure 5 Survival by P53 ( - strong; - weak).

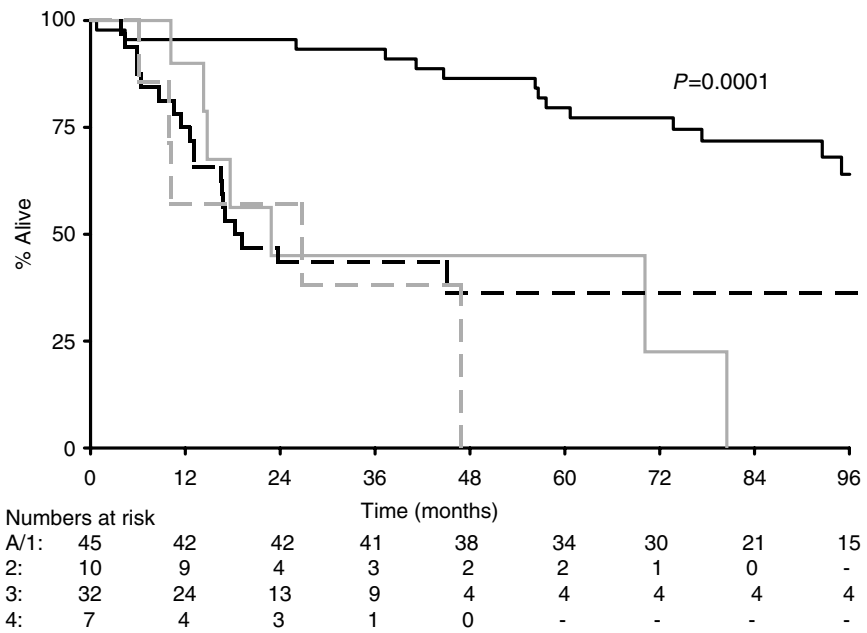

Figure 6 Survival by stage (- stage $\mathrm{A} / \mathrm{I}$; — stage $2 ;$ - - stage 3 ; - stage 4).

$P=0.0001$ ) (Figure 6) and grade (grade $1 v s$ grade $2 v s$ grade 3: 111, 128 and $45 \mathrm{~m}$, respectively, $P=0.02$ ) (Figure 7).

\section{Cox regression on survival}

Cox regression showed stage (Ta or $\mathrm{T} 1 v s \mathrm{~T} 2-4)$ to be the most important independent prognostic factor $(P<0.0001, \mathrm{HR}=1.77$,

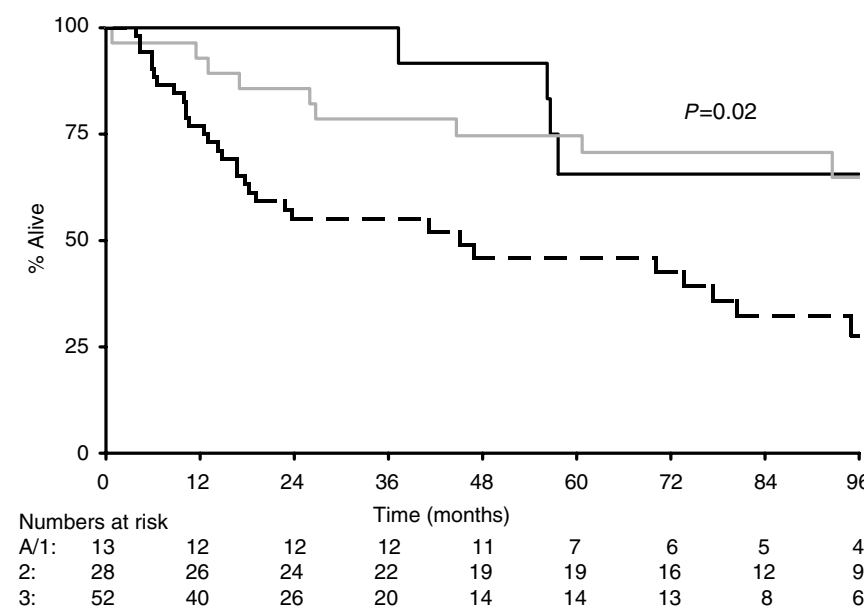

Figure 7 Survival by grade ( - grade I; — grade 2; - - grade 3).

95\% CI $1.34-2.33$ ) and, if it is known, no other variable adds to the accuracy of prognosis. However, if stage is unknown, BAX $(P=0.0003, \quad \mathrm{HR}=3.00, \quad 95 \% \quad \mathrm{CI}=1.64-5.48) \quad$ and $\quad \mathrm{CD} 40 \mathrm{~L}$ $(P=0.04, \mathrm{HR}=1.99,95 \% \mathrm{CI}=1.03-3.83)$ are shown to be important prognostic markers although either of the one rules out the need for the other. No further variables were deemed important in survival prognosis.

\section{DISCUSSION}

A fundamental difficulty in the understanding, prevention and treatment of cancer is that the currently recognised disease classes are each really a collection of diseases having significant features in common (e. g. the organ where the tumour arose), but also many features that distinguish them. The diversity within most disease categories is reflected in a diversity of responses to specific therapeutic regimens. Numerous studies indicate that this clinical heterogeneity reflects underlying molecular heterogeneity, and therefore we have begun to use gene expression measurements to build a higher resolution and more clinically relevant taxonomy of human tumours. Our study analysed a large collection of archived tumour samples, where the treatment history and outcomes of the patients were well documented, and reveals interesting associations with a group of genes involved in regulation of apoptosis.

The CD40 receptor is expressed in many immune cell types and is known to play a central role in both humoral and T-cellmediated immunity, a subject of intense research interest in recent years. It is also expressed on a variety of carcinomas and may therefore be of biological significance in the development and treatment of cancer (Young et al, 1998; Gallagher et al, 2002). Our group has previously examined expression of CD40 by immunohistochemistry in TCC of the bladder and the correlation with known prognostic markers and clinical outcome (Cooke et al, 1999). Seventy-eight percent of the tumours were CD40 positive, with a highly significant association with both lower stage and lower grade $(P<0.001)$. CD40 expression was not found to be an independent prognostic marker of survival in our previous study. We extended these previous observations by additionally assessing the expression of CD40L and P53 in this group of patients to further investigate their role as prognostic markers.

In this study, we were able to demonstrate expression of CD40L in bladder tumour tissue (Figure 8), the first such demonstration in nonlymphoid tissue. Furthermore, overexpression of CD40L was significantly associated with improved outcome (Figure 2). Although overexpression of CD40 was not prognostically significant at the $5 \%$ level $(P=0.12$, Figure 3$)$, it was significantly 

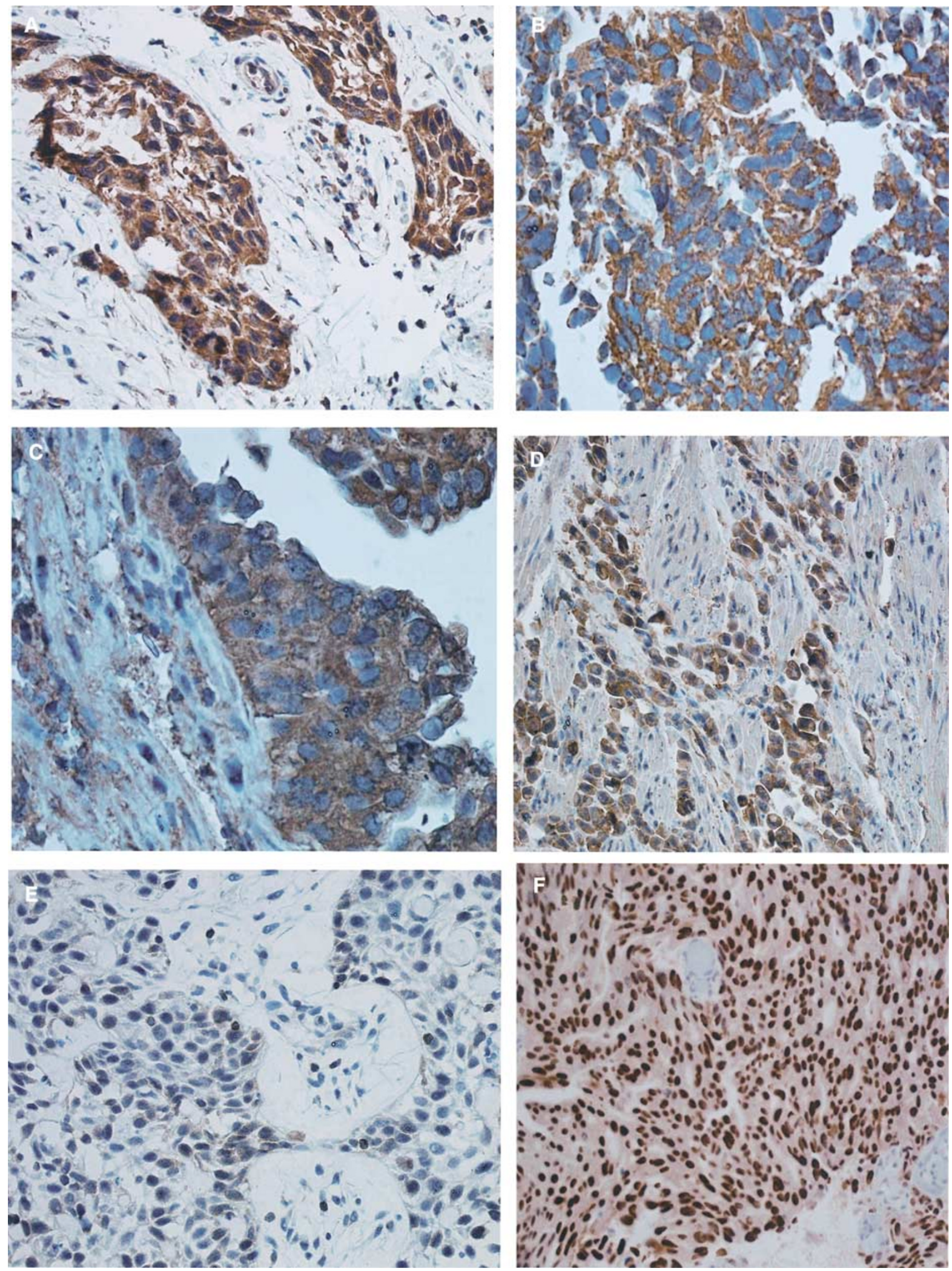

Figure 8 Pathology image. (A) Magnification $\times 40$ objective, CD40 shows diffuse strong cytoplasmic staining in myo invasive transitional cell carcinoma. (B) $\times 40$ objective, CD40L antibodies stains neoplastic cells strongly positive. (C) $\times 40$ objective, BAX shows strong positive staining of the surface in this transitional cell carcinoma. (D) $\times 20$ objective, shows myo invasive islands stain strongly positive for BAX. (E) $\times 20$ objective, weak and focal positivity of BCL2, few tissue lymphocytes serve as positive internal controls. $(\mathbf{F}) \times 20$ objective, strong diffuse nuclear positivity is seen on p53 staining. 
associated with expression of CD40L by Fisher's exact test, suggesting that both ligand and receptor are likely to be necessary for biological effect.

The BCL2 oncoprotein inhibits apoptosis. As a complex network of regulatory pathways control apoptosis and the sensitivity of apoptosis is the result of the balance between pro- and antiapoptotic proteins, we set out to examine other apoptosisregulating proteins. There is increasing evidence from other tumour sites showing the role of BAX as a prognostic marker. In ovarian cancer, it was shown that high BAX expression was associated with significant improvement of the percentage of complete remissions after first-line chemotherapy with Paclitaxel and a platinum analogue. Survival was also high in groups with high BAX expression (Tai et al, 1998). In patients with breast cancer, loss of BAX immunostaining was associated with a decreased response to chemotherapy and shorter survival (Krajewski et al, 1995). In diffuse aggressive non-Hodgkin's lymphoma, reduced BAX expression was associated with a lower 8-year survival (Gascoyne et al, 1997). BAX plays a central role in regulation and commitment to programmed cell death. BAX counteracts the apoptosis-preventing effect of BCL2 and may directly induce apoptosis (Xiang et al, 1996; Zha and Reed, 1997). The proapoptotic BAX is located in the outer mitochondrial membrane (Schlesinger et al, 1997). BAX overexpression induces mitochondrial permeability transition, which leads to the release of cytochrome $c$ (Pastorino et al, 1998). BAX-negative tumours, therefore, may be less susceptible to apoptosis. BAX/P53 pathway analysis in colorectal cancer with hepatic metastases showed that patients with high BAX protein expression in resected liver lesions had a median survival of $53.6 \mathrm{~m}$ compared with $35.4 \mathrm{~m}$ for patients with low BAX expression $(P<0.05)$. Low BAX expression was an independent negative prognostic marker in multivariate regression analysis for all patients independent of the P53 status (Sturm et al, 1999). In our study, overexpression of BAX was associated with a favourable outcome (Figure 1). Expression of BAX was shown to be independent of both BCL2 and P53 both by Fisher's exact test and by Correspondence Analysis, suggesting that it relates to different aspects of the biology of the tumour. On the other hand, BAX expression was associated with expression of CD40 and CD40L, suggesting that all three are linked in their behaviours in this tumour type.

Our results also reveal a significant association between overexpression of CD40 ligand and survival. CD40 ligation thus could be used to sensitise a tumour to conventional treatment modalities such as chemo- or radiotherapy and additionally with a potential for stimulating natural immunity against solid tumours in the manner, which has already been seen in other malignancies both in vitro and in vivo (Eliopoulos et al, 1996; Bergamo et al, 1997; Dilloo et al, 1997; Kikuchi and Crystal, 1999). There is evidence to support the notion of CD40 ligation-induced apoptosis as a mechanism for eliminating neoplastically transformed urothelial cells (Bugajska et al, 2002). A phase I study of recombinant Human $\mathrm{CD} 40 \mathrm{~L}$ in patients with advanced solid tumours or intermediate- or high-grade non-Hodgkin's lymphoma

\section{REFERENCES}

Apakama I, Robinson MC, Walter NM, Charlton RG, Royds JA, Fuller CE, Neal DE, Hamdy FC (1996) Bcl-2 overexpression combined with p53 protein accumulation correlates with hormone-refractory prostate cancer. Br J Cancer 74(8): 1258-1262

Armitage RJ, Fanslow WC, Strockbine L, Sato TA, Clifford KN, Macduff BM, Anderson DM, Gimpel SD, Davis-Smith T, Maliszewski CR et al (1992) Molecular and biological characterization of a murine ligand for CD40. Nature 357: $80-82$

Banchereau J, Bazan F, Blanchard D, Briere F, Galizzi JP, van Kooten C, Liu YJ, Rousset F, Saeland S (1994) The CD40 antigen and its ligand. Annu Rev Immunol 12: 881 - 922 demonstrated encouraging antitumour activity, including a longterm complete remission (Vonderheide et al, 2001). Therapeutic use of CD40L in future will be incorporated into radical treatment of bladder cancer to get a clear picture of their efficacy and toxicity. We intend to use more sophisticated techniques including microarrays in a group of patients undergoing treatment in a randomised setting, with the ultimate hope of being able to further sub-classify bladder tumours into clinically homogenous groups.

Expression of these genes was seen across the range of grades and stages of disease. Once these are stratified into various stages and grades, the number of cases decreases and the statistical power to discriminate is lost. It would be very interesting to follow patients with low-grade tumours at diagnosis in a randomised clinical trial setting with Bax, Bcl-2, CD40 and CD40L mapping and note whether overexpression or underexpression of these markers by immunohistochemistry predates the aggressive clinical behaviour and therefore indicates a point of therapeutic intervention.

In conclusion, this is the first study that has examined expression of BAX in all stages of transitional cell carcinoma of the bladder. We believe that analysis of molecular markers such as BAX, BCL2, CD40 and CD40L may prove valuable in identifying patients with poor prognosis bladder cancers who may benefit from aggressive treatment and this should be considered in future trial design. Bladder is a particularly attractive model to study, as it provides a conveniently isolated field to test new therapies, and repeated endoscopic assessment and tissue biopsies are possible during the course of treatment and post-treatment to evaluate the response to the therapy. Furthermore, the disease itself, if advanced to muscle invasion, carries a poor outlook as seen in our series, with median survival dropping from $128 \mathrm{~m}$ for stage $\mathrm{Ta}$ or T1, to $23 \mathrm{~m}$ for stage $\mathrm{T} 2,19 \mathrm{~m}$ for stage T3 and $27 \mathrm{~m}$ for stage T4 with a $P$-value of 0.0001 (Figure 6). This also strengthens the statement that $\mathrm{Ta}, \mathrm{T} 1$ and invasive bladder cancers are two different entities with remarkably different clinical outcomes.

Endoscopic assessment of bladder tissue at various time points in organ preservation strategies will also help us to understand and answer many aspects of programmed cell death. All forms of cancer therapy risk selecting for resistance, a problem compounded by plasticity of the tumour cell genome (Green and Evan, 2002). The most effective strategy will definitely be a combination treatment attacking several targets specific to cancer. The evolution of such sophisticated forms of treatment will undoubtedly proceed by a process that employs both rational and empiric approaches. Our study generates a hypothesis linking proapoptotic and antiapoptotic markers to overall survival and needs to be tested robustly in a randomised clinical trial setting.

\section{ACKNOWLEDGEMENTS}

We are grateful to Mrs Irene Davies and her family for their generous donation in support of this research.
Bergamo A, Bataille R, Pellat-Deceunynck C (1997) CD40 and CD95 induce programmed cell death in the human myeloma cell line XG2. $\mathrm{Br} J$ Haematol 97: 652-655

Bronner MP, Culin C, Reed JC, Furth EE (1995) The BCL2 proto-oncogene and the gastrointestinal epithelial tumour progression model. Am J Pathol 146(1): 20-26

Bugajska U, Georgopoulos N, Southgate J, Johnson PW, Graber P, Gordon J, Selby PJ, Trejdosiewicz LK (2002) The effects of malignant transformation on susceptibility of human urothelial cells to CD40mediated apoptosis. JNCI 94(8): 1381-1385 
Cooke PW, James ND, Ganesan R, Wallace M, Burton A, Young LS (1999) CD40 expression in bladder cancer. J Pathol 188(1): $38-43$

Cooke PW, James ND, Ganesan R, Burton A, Young LS, Wallace DM (2000) Bcl-2 expression identifies patients with advanced bladder cancer treated by radiotherapy who benefit from neoadjuvant chemotherapy. Br J Urol Int 85(7): 829-835

Cox DR (1972) Regression models and life tables. J Roy Stat Soc B 34: $187-220$

Dilloo D, Brown M, Roskrow M, Zhong W, Holladay M, Holden W, Brenner M (1997) CD40 ligand induces an antileukemia immune response in vivo. Blood 90: 1927 - 1933

Eliopoulos AG, Dawson CW, Mosialos G, Floettmann JE, Rowe M, Armitage RJ, Dawson J, Zapata JM, Kerr DJ, Wakelam MJ, Reed JC, Kieff E, Young LS (1996) CD40-induced growth inhibition in epithelial cells is mimicked by Epstein-Barr virus-encoded LMP1: involvement of TRAF3 as a common mediator. Oncogene 13: 2243-2254

Freiha F, Reese J, Torti FM (1996) A randomized trial of radical cystectomy versus radical cystectomy plus cisplatin, vinblastine and methotrexate chemotherapy for muscle invasive bladder cancer. J Urol 155(2): 495- 499

Gallagher NJ, Eliopoulos AG, Agathangelo A, Oates J, Crocker J, Young LS (2002) CD40 activation in epithelial ovarian carcinoma cells modulates growth, apoptosis and cytokine secretion. Mol Pathol 55: 110-120

Gallo O, Chiarelli I, Boddi V, Bocciolini C, Bruschini L, Porfirio B (1999) Cumulative prognostic value of p53 mutations and BCL2 protein expression in head-and-neck cancer treated by radiotherapy. Int $J$ Cancer 84(6):573-579

Gascoyne RD, Krajewska M, Krajewski S, Connors JM, Reed JC (1997) Prognostic significance of BAX protein expression in diffuse aggressive non-Hodgkin's lymphoma. Blood 90: 3173-3178

Green RD, Evan GI (2002) A matter of life and death. Cancer Cell 1: 19-30

Greenacre MJ (1984) Theory and Applications of Correspondence Analysis. London: Academic Press

Grewal IS, Flavell RA (1998) CD40 and CD154 in cell-mediated immunity. Ann Rev Immunol 16: 111 - 135

Harima Y, Harima K Shikata N, Oka A, Ohnishi T, Tanaka Y (1998) BAX and Bcl-2 expressions predict response to radiotherapy in human cervical cancer. J Cancer Res Clin Oncol 124(9): 503-510

Hellemans P, van Dam PA, Weyer J, van Oosterom AT, Buytaert P, Van Marck E (1995) Prognostic value of BCL2 expression in invasive breast cancer. Br J Cancer 72(2): 354-360

Henn V, Slupsky JR, Grafe M, Anagnostopoulos I, Forster R, MullerBerghaus G, Kroczek RA (1998) CD40 ligand on activated platelets triggers an inflammatory reaction of endothelial cells. Nature 391: $591-594$

Hussain SA, Moffitt DD, Glaholm JG, Peake D, Wallace DMA, James ND (2001) A phase I/II study of toxicity and response in patients receiving synchronous chemoradiotherapy for locally advanced bladder cancer. Ann Oncol 12(7): 929-935

International Collaboration of Trialists (1999) Neoadjuvant cisplatin, methotrexate and vinblastine chemotherapy for muscle invasive bladder cancer: a randomised controlled trial. Lancet 354(9178): 533-540

Kaplan EL, Meier P (1958) Non parametric estimation from incomplete observations. J Am Stat Assoc 53: 457-481

Kikuchi T, Crystal RG (1999) Anti-tumour immunity induced by in vivo adenovirus vector-mediated expression of CD40 ligand in tumour cells. Human Gene Ther 10: 1375 - 1387.

King ED, Matteson J, Jacobs SC, Kyprianou N (1996) Incidence of apoptosis, cell proliferation and BCL2 expression in transitional cell carcinoma of the bladder: association with tumour progression. J Uro 155(1): $316-320$

Kong G, Shin KY, Oh YH, Lee JJ, Park HY, Woo YN, Lee JD (1998) Bcl-2 and p53 expressions in invasive bladder cancers. Acta Oncol 37(7-8): $715-720$

Krajewska M, Krajewski S, Epstein JI, Shabaik A, Sauvageot J, Song K, Kitada S, Reed JC (1996) Immunohistochemical analysis of BCL2, BAX bcl-X, and mcl-1 expression in prostate cancers. Am J Pathol 148(5): $1567-1576$
Krajewski S, Blomqvist C, Franssila K, Krajewska M, Wasenius VM, Niskanen E, Nordling S, Reed JC (1995) Reduced expression of proapoptotic gene BAX is associated with poor response rates to combination chemotherapy and shorter survival in women with metastatic breast adenocarcinoma. Cancer Res 55: 4471-4478

Lee E, Park I, Lee C (1997) Prognostic markers of intravesical bacillus Calmette-Guerin therapy for multiple, high-grade, stage T1 bladder cancers. Int J Urol 4(6): $552-556$

Malmstrom PU, Rintala E, Wahlqvist R, Hellstrom P, Hellsten S, Hannisdal E (1996) Five-year follow-up of a prospective trial of radical cystectomy and neoadjuvant chemotherapy: Nordic Cystectomy Trial I. J Urol 155(6): $1903-1906$

Pastorino JG, Chen ST, Tafani M, Snyder JW, Farber JL (1998) The overexpression of BAX produces cell death upon induction of the mitochondrial permeability transition. J Biol Chem 273: $7770-7775$

Peto R, Pike MC, Armitage P, Breslow NE, Cox DR, Howard SV, Mantel N, McPherson K, Peto J, Smith PG (1977) Design and analysis of randomised clinical trials requiring prolonged observation of each patient II. Analysis and example. Br J Cancer 35: 1-39

Pollack A, Wu CS, Czerniak B, Zagars GK, Benedict WF, McDonnell T] (1997) Abnormal BCL2 and pRb expression are independent correlates of radiation response in muscle-invasive bladder cancer. Clin Cancer Res 3(10): $1823-1829$

Schlesinger PH, Gross A, Yin XM, Yamamoto K, Saito M, Waksman G, Korsmeyer SJ (1997) Comparison of the ion channel characteristics of proapoptotic BAX and antiapoptotic BCL-2. Proc Natl Acad Sci USA 94: $11357-11362$

Shiina H, Igawa M, Urakami S, Honda S, Shirakawa H, Ishibe T (1996) Immunohistochemical analysis of BCL2 expression in transitional cell carcinoma of the bladder. J Clin Pathol 49(5): 395-399

Spriggs MK, Armitage RJ, Strockbine L, Clifford KN, Macduff BM, Sato TA, Maliszewski CR, Franslow WC (1992) Recombinant human CD40 ligand stimulates B cell proliferation and immunoglobulin E secretion. $J$ Exp Med 176: 1543 - 1550

Stockle M, Wellek S, Meyenburg W, Voges GE, Fischer U, Gertenbach U, Thuroff JW, Huber C, Hohenfellner R (1996) Radical cystectomy with or without adjuvant polychemotherapy for non-organ-confined transitional cell carcinoma of the urinary bladder: prognostic impact of lymph node involvement. Urology 48(6): $868-875$

Sturm I, Kohne C, Wolff G, Petrowsky H, Hillebrand T, Hauptmann S, Lorenz M, Dorken B, Daniel PT (1999) Analysis of the P53/BAX pathway in colorectal cancer: low BAX is a negative prognostic factor in patients with resected liver metastases. J Clin Oncol 17(5): 1364-1374.

Tai YT, Lee S, Niloff E, Weisman C, Strobel T, Cannistra SA (1998) BAX protein expression and clinical outcome in epithelial ovarian cancer. $J$ Clin Oncol 16: 2583-2590 [see comments]

Vonderheide RH, Dutcher JP, Anderson JE, Eckhardt SG, Stephans KF, Razvillas B, Garl S, Butine MD, Perry VP, Armitage RJ, Ghalie R, Caron DA, Gribben JG (2001) Phase I study of recombinant human CD40 ligand in cancer patients. J Clin Oncol 3280-3287

Wallace DM, Raghavan D, Kelly KA, Sandeman TF, Conn IG, Teriana N, Dunn J, Boulas J, Latief T (1991) Neo-adjuvant (pre-emptive) cisplatin therapy in invasive transitional cell carcinoma of the bladder. $B r$ J Uro 67: $608-615$

Xiang J, Chao DT, Korsmeyer SJ (1996) BAX-induced cell death may not require interleukin 1 beta-converting enzyme-like proteases. Proc Nat Acad Sci USA 93: $14559-14563$

Ye D, Li H, Qian S, Sun Y, Zheng J, Ma Y (1998) BCL2/BAX expression and p53 gene status in human bladder cancer: relationship to early recurrence with intravesical chemotherapy after resection. J Urol 160(6, part 1): $2025-2028$

Young LS, Eliopoulos AG, Gallagher NJ, Dawson CW (1998) CD40 and epithelial cells: across the great divide. Immunol Today 19: 502-506

Zha H, Reed JC (1997) Heterodimerization-independent functions of cell death regulatory proteins $\mathrm{BAX}$ and $\mathrm{Bcl}-2$ in yeast and mammalian cells. $J$ Biol Chem 272: $31482-31488$ 